\title{
Harnessing RNA interference to prevent or treat HSV-2 and HIV Judy Lieberman*
}

Address: CBR Institute for Biomedical Research, Harvard Medical School, Boston, Massachusetts 02115, USA

* Corresponding author

from 2006 International Meeting of The Institute of Human Virology

Baltimore, USA. 17-2I November, 2006

Published: 21 December 2006

Retrovirology 2006, 3(Suppl I):S48 doi:I0.I I86/I742-4690-3-SI-S48

() 2006 Lieberman; licensee BioMed Central Ltd.

Harnessing RNAi presents an opportunity for treating or preventing viral disease. The main obstacle is delivering siRNAs into the cytosol of target cells in vivo. Topical delivery at mucosal surfaces may be especially effective. Herpes simplex virus 2 (HSV-2) infection causes significant morbidity and is an important cofactor for transmission of HIV infection. We investigated whether intravaginal application of siRNAs could protect mice from lethal herpes simple virus type 2 (HSV-2) vaginal infection. siRNAs mixed with lipid are efficiently taken up by epithelial and lamina propria cells and silence gene expression in the mouse vagina and ectocervix for at least 9 days. Intravaginal application of siRNAs targeting the HSV-2 UL27 and UL29 genes, encoding an envelope glycoprotein and a DNA binding protein, respectively, was well tolerated, did not induce interferon responsive genes or cause inflammation, and protected mice when administered before and/or following lethal HSV-2 challenge. These results suggest that siRNAs are attractive candidates for the active component of an antiviral microbicide. We are currently working to see if we can use this approach to prevent HIV transmission. We have also developed a method using antibody-protamine fragment fusion proteins to deliver siRNAs systemically and specifically to achieve silencing in primary cells bearing a targeted receptor. Potential applications of this strategy for treating HIV infection will be discussed. 